BRAVZULIAN JOURNAL

OF MEDICAL AND BIOLOGICAL RESH.ARCH

www.bjournal.com.br
ISSN 0100-879X

Volume 44 (6) 497-605 June 2011

BIOMEDICAL SCIENCES

AND

CLINICAL INVESTIGATION

Braz J Med Biol Res, June 2011, Volume 44(6) 514-523

doi: 10.1590/S0100-879X2011007500054

Essential regulatory elements for NHE3 gene transcription in renal proximal tubule cells

E.A. Neri, C.N.A. Bezerra and N.A. Rebouças

The Brazilian Journal of Medical and Biological Research is partially financed by

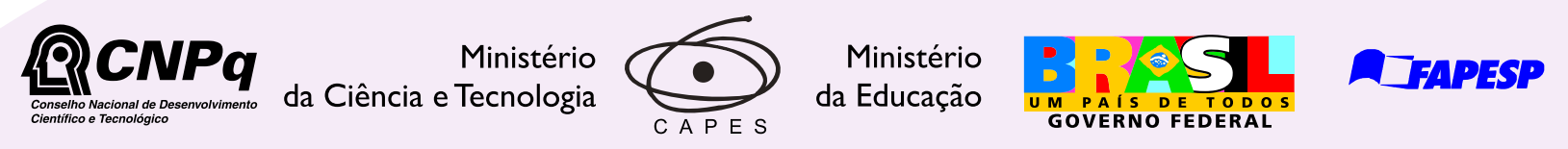

Institutional Sponsors
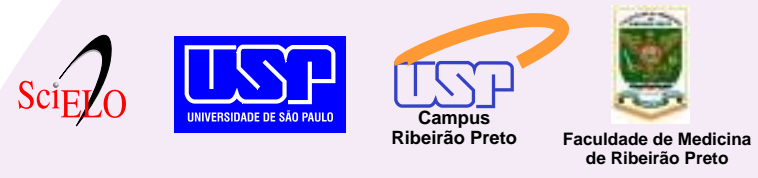
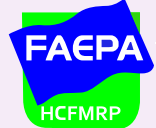

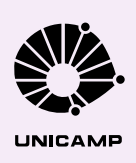

Ф SHIMADZU

GE Healthcare
Hotsite of proteomics metabolomics developped by:

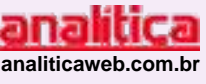

Thermo SCIENTIFIC 


\title{
Essential regulatory elements for NHE3 gene transcription in renal proximal tubule cells
}

\author{
E.A. Neri, C.N.A. Bezerra and N.A. Rebouças
}

Departamento de Fisiologia e Biofísica, Instituto de Ciências Biomédicas, Universidade de São Paulo, São Paulo, SP, Brasil

\begin{abstract}
The objectives of the present study were to identify the cis-elements of the promoter absolutely required for the efficient rat NHE3 gene transcription and to locate positive and negative regulatory elements in the 5'-flanking sequence (5'FS), which might modulate the gene expression in proximal tubules, and to compare this result to those reported for intestinal cell lines. We analyzed the promoter activity of different 5'FS segments of the rat NHE3 gene, in the OKP renal proximal tubule cell line by measuring the activity of the reporter gene luciferase. Because the segment spanning the first $157 \mathrm{bp}$ of $5^{\prime} \mathrm{FS}$ was the most active it was studied in more detail by sequential deletions, point mutations, and gel shift assays. The essential elements for gene transcription are in the region -85 to -33 , where we can identify consensual binding sites for Sp1 and EGR-1, which are relevant to NHE3 gene basal transcription. Although a low level of transcription is still possible when the first 25 bp of the 5'FS are used as promoter, efficient transcription only occurs with $44 \mathrm{bp}$ of $5^{\prime} \mathrm{FS}$. There are negative regulatory elements in the segments spanning -1196 to -889 and -467 to -152 , and positive enhancers between -889 and -479 bp of 5 'FS. Transcription factors in the OKP cell nuclear extract efficiently bound to DNA elements of rat NHE3 promoter as demonstrated by gel shift assays, suggesting a high level of similarity between transcription factors of both species, including Sp1 and EGR-1.
\end{abstract}

Key words: $\mathrm{Na}^{+} / \mathrm{H}^{+}$exchanger isoform 3; Gene promoter; Specificity protein 1; Early growth response factor 1; Proximal tubules

\section{Introduction}

$\mathrm{NHE3}$ is an $\mathrm{Na}^{+} / \mathrm{H}^{+}$exchanger member of the SLC9A family of transporters and is the predominant route for $\mathrm{H}^{+}$ secretion in the apical membrane of proximal tubules, where it plays a key role in fluid reabsorption and $\mathrm{pH}$ homeostasis (1). Regulation of NHE3 activity, which is relevant for homeostatic mechanisms, depends on a highly complex interplay of proteins and signaling pathways, which adjust not only the $\mathrm{Na}^{+}$reabsorption and $\mathrm{H}^{+}$secretion, but also the transepithelial flux of other solutes reabsorbed by transporters indirectly coupled to NHE3 $(2,3)$. In parallel to acute regulatory mechanisms of NHE3 activity, the expression of this protein is also adjusted to different chronic physiological or pathological conditions. Chronic metabolic acidosis (4-6), reduced extracellular volume (7), exposure to angiotensin II $(8,9)$, insulin (10), glucocorticoids (10-12), thyroid (13), or parathyroid hormones $(14,15)$ are all conditions that can alter NHE3 expression. The regulated steps that result in changes of NHE3-mRNA levels in these different states can occur at any point from gene transcription to the final mRNA degradation in the cytoplasm.
The rat NHE3 promoter region was first sequenced in 1996 by Cano (16) and by Kandasamy and Orlowski (12). A predominant initiation site was identified at position -97 (thymine) in kidney and intestine, although multiple start sites were clustered between nucleotides -100 and -96 relative to the translation initiation site (12). Comparing the $1.6 \mathrm{~kb}$ of the 5'-flanking sequence (5'FS) of rat and human NHE3 promoter, it was possible to detect $39 \%$ overall identity. Higher identity, 79\%, was detected in the first $100 \mathrm{bp}$, where specificity protein $1 / 3(\mathrm{Sp} 1 / 3)$, early growth response factor 1 (EGR-1), activator protein-2 (AP2), and TATA-like sequences appear at the same positions in both promoters (17). Hormone receptor-responsive elements (e.g., triiodothyronine and glucocorticoid receptor binding sites) were identified in less restricted positions and orientation in the NHE3 gene promoters of these two species. Human and rat NHE3 gene promoters were analyzed in detail in the intestinal cell line Caco-2. Some relevant elements that modulate gene transcription upon stimulation with phorbol 12-myristate 13-acetate (PMA), sodium butyrate (NaB), or

Correspondence: N.A. Rebouças, Departamento de Fisiologia e Biofísica, Instituto de Ciências Biomédicas, USP, Av. Professor Lineu Prestes, 1524, 05508-000 São Paulo, SP, Brasil. Fax: +55-11-3091-7285. E-mail: nancy@icb.usp.br

Received October 24, 2010. Accepted April 11, 2011. Available online May 2, 2011. Published June 13, 2011. 
interferon- $y /$ tumor necrosis factor- $\alpha$ (INF- $y / T N F-\alpha)$ were mapped to the proximal promoter, where the transcription factors Sp1, Sp3 and EGR-1 can bind and compete for neighboring binding sites (18-20).

Our main goal in the present study was to analyze the transcriptional regulation of the NHE3 gene in renal proximal tubule cells and to compare our observations to those reported in previous studies on the regulation of this gene in intestinal cells. We report a detailed analysis of the NHE3 promoter in a proximal tubule cell line of opossum kidney (OKP). We focused on identifying stimulatory or inhibitory regions within the first 2000 bp of the 5'FS of NHE3 gene, and located the relevant regulatory cis-elements in the proximal promoter.

\section{Material and Methods}

We purchased Dulbecco's modified Eagle's medium (DMEM), heat-inactivated fetal bovine serum, sodium piruvate, and penicillin/streptomycin from Life Technologies (Gibco BRL, USA); Lipofectamine Plus Reagent and Platinum Taq DNA Polymerase from Invitrogen (USA); Dual Luciferase Assay System, pGL3-basic vector and $\mathrm{pRL}-\mathrm{CMV}$ vector, and endonuclease restriction enzymes from Promega (USA). OKP cells, a clonal sub-line of the opossum (Didelphis virginiana) kidney cell line originally described by Cole et al. (21), were kindly provided by Dr. Orson W. Moe (University of Texas Southwestern Medical Center, Dallas, TX, USA). All other reagents and chemicals were obtained from Sigma Chemical (USA), unless otherwise specified.

\section{Cell culture}

OKP cells were maintained in $75-\mathrm{cm}^{2}$ tissue culture flasks in DMEM containing $10 \%$ heat-inactivated fetal bovine serum, $1 \mathrm{mM}$ sodium piruvate, $100 \mathrm{U} / \mathrm{mL}$ penicillin, and $100 \mu \mathrm{g} / \mathrm{mL}$ streptomycin. Cultures were incubated at $37^{\circ} \mathrm{C}$ in a humidified $5 \% \mathrm{CO}_{2}$ air atmosphere.

\section{Promoter luciferase constructs}

We obtained by PCR a segment of the rat NHE3 gene promoter by using primers with endonuclease restriction sites at 5'-ends (forward primer: TCTAGAAAGTAAGCC CCACCTGAG; reverse primer: AGGAGCCGACACGCATA CCGTG). The PCR product was cloned and sequenced in both directions. Using restriction enzymes, we obtained 2 different segments of the promoter, -2095/-17 and -2095/+55, which were cloned in pGL3-basic vector (Promega). The inserted fragment containing $2150 \mathrm{bp}$, which encompasses $2095 \mathrm{bp}$ from the 5'FS and $55 \mathrm{bp}$ from the first exon of the rat NHE3 gene, was used for subsequent deletions of the NHE3 gene 5'-flanking region, keeping the common 3'-end at $+55 \mathrm{bp}$. The different segments of the NHE3 gene regulatory region were generated by using Bsu36I and one of the following restriction endonucleases: Pvull (-1196/+55); Nsil (-889/+55); Sstll (-467/+55) and Apal (-152/+55). After digestion, the constructs were purified, blunt ended and religated into the pGL3 vector. Since we could not obtain smaller segments with restriction enzymes, we obtained them by PCR amplification with the primers depicted in Table 1. These reactions resulted in the following segments: $-157 /+31,-85 /+31,-65 / 31$. Restriction sites were added to the 5 '-end of the primers for directional cloning in the pGL3basic vector. Segments corresponding to $-44 /+31,-33+31$, and $-25 /+31$ were obtained by annealing of the synthetic sense and antisense corresponding oligonucleotides (Table 1 ), and were inserted into the same gene reporter expression vector, pGL3-basic.

\section{Point mutations in putative regulatory elements}

Mutant constructs were obtained by site-directed mutagenesis in the forward or reverse PCR primers (Table 2).

Table 1. Nucleotide sequences of forward and reverse primers used to obtain segments of the $5^{\prime}$-flanking sequence of the rat NHE3 gene.

\begin{tabular}{lll}
\hline Region & Forward & Reverse \\
\hline$-25 /+31$ & ACAGCAGGTACCGGCCACCCGGGCAGG & TGAGCAAAGCTTCTCTGCGCTATCCCGGAGCTGG \\
& CCTGGGGACCTGACTGCTGCCAGCTCCG & CAGCAGTCAGGTCCCCAGGCCTGCCCGGGTGGCC \\
& GGATAGCGCAGAGAGCTTTCTCA & GGTACCTGCTGT \\
$-33 /+31$ & ACAGCAGGTACCGATTAAAGGCCACCC & TGAGCAAAGCTTCTCTGCGCTATCCCGGAGCTGGCA \\
& GGGCAGGCCTGGGGACCTGACTGCTGCC & GCAGTCAGGTCCCCAGGCCTGCCCGGTGGCCTTT \\
& AGCTCCGGGATAGCGCAGAGAAGCTTTGCTCA & AATCGGTACCTGCTGT \\
$-44 /+31$ & ACAGCAGGTACCGAGGGGGCAGCGGGATTAAA & TGAGCAAAGCTTCTCTGCGCTATCCCGGAGCTGGCA \\
& GGCCACCCGGGCAGGCCTGGGACCTGACTGC & GCAGTCAGGTCCCCAGGCCTGCCCGGTGGCCTTTA \\
& TGCCAGCTCCGGGATAGCGAGAGAAGCTTTGCACA & ATCCCGCTGCCCCTCGGTACCTGCTGT \\
$-65 /+31$ & AGCAGGTACCAAGGCCCCGCCCTG & AGCAAAGCTTCTCTGCGCTATCCCGGAG \\
$-85 /+31$ & AGCAGGTACCACGTGCGCAGCGGG & AGCAAAGCTTCTCTGCGCTATCCCGGAG \\
$-157 /+31$ & AGCAGGTACCGGGGCCCAACCAAGTAGGT & AGCAAAGCTTCTCTGCGCTATCCCGGAG \\
\hline
\end{tabular}

Sites for restriction enzymes are given in bold. 
Table 2. Sequence of primers used to obtain segments with punctual mutations in putative regulatory elements.

\begin{tabular}{|c|c|c|}
\hline Region & Forward & Reverse \\
\hline$-80 /+31$ (Sp1) & AGCAGGTACCCGCAGCGGGG $\underline{A}$ CGGGAAG & AGCAAAGCTTCTCTGCGCTATCCCGGAG \\
\hline$-80 /+31(E G R-1)$ & AGCAGGTACCCGCAGCGG $\underline{A}$ G $\underline{A} C G G G A A G$ & AGCAAAGCTTCTCTGCGCTATCCCGGAG \\
\hline$-60 /+31(\mathrm{Sp} 1)$ & AGCAGGTACCCCCAACCCTGGCGGGA & AGCAAAGCTTCTCTGCGCTATCCCGGAG \\
\hline$-60 /+31(\mathrm{Sp} 1 / \mathrm{AP} 2)$ & AGCAGGTACCCCCAACCCTGAAGCGGGA & AGCAAAGCTTCTCTGCGCTATCCCGGAG \\
\hline
\end{tabular}

Sites for restriction enzymes are given in bold and punctual mutations are italicized and underlined.

The following promoter segments were obtained: -80 to +31 mutated at position -72 ( $G$ to $A$; a putative binding site for EGR-1/Sp1); -80 to +31 mutated at positions -72 and -70 ( $G$ to $A$ ); -60 to +31 mutated at positions -58 and -57 (CG to AA; a putative binding site for Sp1); -60 to +31 mutated at positions -57 and -58 (CG to AA) and -51 and -50 (GC to $A A$; sites for Sp1 and EGR-1/AP2, respectively).

\section{Plasmid construct purification and quantification}

Plasmids were purified in maxi-prep spin columns (Wiz$\operatorname{ard}^{\circledR}$ DNA Purification System, Promega) and quantified by spectrometry and by agarose gel electrophoresis. On agarose gels, the DNA bands were dyed with SYBR green I (Molecular Probes, USA), quantified with a laser densitometer (STORM - Molecular Dynamics, USA) and compared to a mass ladder using the Image Quant 5.1 software.

\section{Luciferase reporter gene assay}

OKP cells, $70-80 \%$ confluent, were transiently cotransfected with $400 \mathrm{ng}$ pGL3-basic vector containing chimerical NHE3 promoter gene constructs or promoterless pGL3-basic vector, and 4 ng of the internal control pRLCMV vector (Promega), which contains Renilla luciferase downstream of the CMV promoter, using Lipofectamine Plus Reagent (Invitrogen) according to the manufacturer protocol. The transfection medium was left to stand for 4 $\mathrm{h}$ and the cells were then incubated in fresh medium with serum for an additional $24 \mathrm{~h}$. Luciferase was analyzed using the Dual-Luciferase Reporter Assay System (Promega).

\section{Preparation of nuclear extracts and electrophoretic mobility shift assay (EMSA)}

Nuclear extracts were prepared using the Nuclear Extraction Kit (Chemicon, USA) according to manufacturer instructions. All oligonucleotides for EMSA were synthesized by Invitrogen Life Technologies. Complementary oligonucleotides were rendered double stranded by heating to $100^{\circ} \mathrm{C}$ for $10 \mathrm{~min}$ and slowly cooling at room temperature. The following probes containing EGR-1/Sp1 (wild type, WT) and Sp1/AP2 (WT) binding sites were used: 5'-CGCAGCGGGGGCGGG AAGGC-3', and 5'-CCCCGCCCTGGCGCGGGAGG-3'. The probes were end-labeled with $\left[\mathrm{Y}^{32} \mathrm{P}\right]-A T P$ obtained from Perkin Elmer (10 pmol consensus oligonucleotides, $15 \mathrm{pmol}\left[\mathrm{Y}^{32}{ }^{32} \mathrm{P}\right]-\mathrm{ATP}$, and 10 units T4 polynucleotide kinase, incubated for $90 \mathrm{~min}$ at $37^{\circ} \mathrm{C}$ ) and were purified with S-300 HR columns (GE Healthcare, UK). Binding reaction mixtures [10 $\mu \mathrm{g}$ nuclear protein of OKP, $1 \mu \mathrm{g}$ poly $(\mathrm{dl}-\mathrm{dC}),{ }^{32} \mathrm{P}$-labeled probe $(80,000 \mathrm{cpm}), 50 \mathrm{mM} \mathrm{NaCl}, 0.2 \mathrm{mM}$ EDTA, $0.5 \mathrm{mM}$ DTT, $2 \%$ glycerol, and $210 \mathrm{mM}$ Tris- $\mathrm{HCl}$ ] were incubated for $20 \mathrm{~min}$ at room temperature. The binding specificity of these complexes was verified by competition assays with unlabeled WT probes in 5-, 10-, 20-, 50-, and 100-fold molar excess or mutated probes in 50-fold molar excess added to the reaction mixture 20 min before the labeled probe. DNA-protein complexes were separated by electrophoresis through a $5.5 \%$ nondenaturing polyacrylamide gel. The gels were vacuum-dried, exposed to a phosphor-imaging screen, and the image was scanned with the Typhoon scanner (Molecular Dynamics) and analyzed using the Image Quant software (GE). The super shift analyses were performed using $3 \mu \mathrm{g}$ anti-Sp1 (Abcam, USA), anti-Sp3 (Abcam) and anti-EGR-1 (Abcam) rabbit polyclonal antibodies incubated with the binding reaction mixtures overnight at $4^{\circ} \mathrm{C}$.

\section{SDS-PAGE and immunoblotting}

Protein samples were solubilized in SDS sample buffer (2\% SDS, $10 \%$ glycerol, $100 \mathrm{mM}$ dithiothreitol, $0.1 \%$ bromophenol blue, $50 \mathrm{mM}$ Tris, $\mathrm{pH} 6.8$ ), and proteins were separated by SDS-PAGE using $7.5 \%$ polyacrylamide gels according to Laemmli (22). Immunoblotting was performed as previously described (23).

\section{Statistical analysis}

Data are reported as means \pm SEM. Comparison between two groups was performed using the unpaired $t$-test. If more than two groups were compared, statistical significance was determined by ANOVA followed by the Tukey post hoc test. A P value $<0.05$ was considered to be statistically significant.

\section{Results}

\section{Functional mapping of the rat NHE3 promoter in OKP cells}

Firstly, we analyzed the promoter efficiency of two different segments of the rat NHE3 gene 5'FS, -2095/+55 and $-2095 /-17$, considering as +1 the major transcription start site described by Kandasamy and Orlowski (12). Although the 
$-2095 /+55$ segment was able to induce transcription seven times above the basal level, no difference was observed between the -2095/-17 segment and the promoterless construct (data not shown). This result, which is in agreement with data reported by Rohman et al. (24), is additional evidence of the correct mapping of the transcription start site and confirms the absolute requirement of this region for transcription initiation.

A series of recombinant plasmids containing different segments of the 5'FS of the rat NHE3 gene were transiently transfected into OKP cells and their relative activity is depicted in Figure 1. The promoter segment -1196/+55 showed no difference in activity from the $-2095 /+55$ region. The segment spanning $-889 /+55$ was three times more efficient as a transcription activator than the previous segments, suggesting the presence of inhibitory elements between -1196 and -889. By removing an additional 422 bp from the 5'-end, we observed a decrease in the expression of the reporter gene, suggesting the presence of putative enhancers of transcription present between -889 and -467 . Further deletion of $315 \mathrm{bp}$ from the 5'-end, which produced the $-152 /+55$ segment, resulted in a $\sim 7$-fold increase in luciferase activity, which was almost 60-fold higher than the promoterless vector. These initial experiments showed us that probably the most important regulators were in this region, what made it necessary to carry out a detailed analysis of the proximal NHE3 promoter, including the first
$150 \mathrm{bp}$ of the 5'FS, a region in which several putative regulatory elements have been identified by Kandasamy and Orlowski (12) and Kiela et al. (25), as shown in Figure 2A.

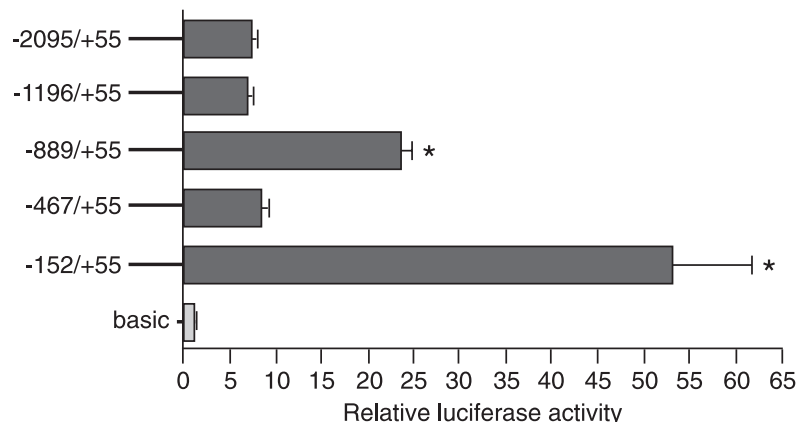

Figure 1. Promoter activity of various 5'-deletion constructs of the rat NHE3 gene promoter region. A series of chimeric deletion mutants of the promoter-Firefly luciferase gene in the parent vector pGL3-basic, with different promoter 5'-ends and a common 3'-end $(+55)$, were cotransfected with $\mathrm{pRL}$ into OKP cells. After $24 \mathrm{~h}$, the cells were lysed and assayed for Firefly and Renilla luciferase activities. The promoter activity is reported relative to the activity of the promoterless vector and is normalized for variations in transfection efficiency using Renilla luciferase activity. Data are reported as means \pm SEM for at least three independent transfection experiments performed in triplicate. The spanning region is indicated on the left side of each bar. ${ }^{*} \mathrm{P}$ $<0.01$, compared to the other constructs and to basic (ANOVA followed by the Tukey test).

\section{A}

B -150 ACCAAGTAGg TGgGCGTGAG CAGCACAGGC GCGAGACCTT CTAACGCAGC

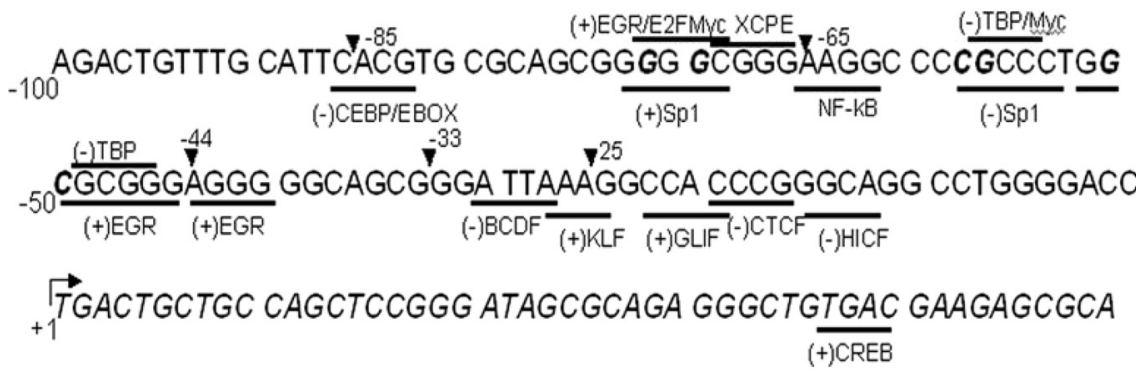

Figure 2. Sequence of the 150 nucleotides of the 5'-flanking region and of 50 nucleotides of the first exon of the rat NHE3 gene. The numbers on the left indicate the position of the nucleotide relative to the major transcription start site. Putative binding sites for various transcription factors are underlined. $A$, The consensus binding sites described by Kandasamy and Orlowski (12), and by Kiela et al. (25) (italic letters); $B$, some of the putative regulatory elements identified by analysis with MatInspector-Genomatix are underlined and identified. The black arrowheads indicate the successive deletions. Nucleotides in bold/italic are the sites of point mutations. 
We submitted the rat NHE3 promoter region to Genomatix for comparison to the Matrix Family Library Version 8.1 (MatInspector library), and some of the identified putative cis-elements are depicted in Figure 2B.

A promoter segment spanning nucleotides -157/+31 was generated by PCR amplification and used for comparison with the promoter activity of the shorter segments also obtained by PCR. The activity of the $-157 /+31$ segment was the same as that spanning nucleotides $152 /+55$. Removal of 72 bp of the 5 '-end $(-85 /+31)$ did not significantly affect the promoter activity, but removal of a further $20 \mathrm{bp}(-65 /+31)$ resulted in a significant reduction in reporter gene expression (Figure 3). Removal of the following 21 nucleotides did not alter the promoter activity that continued to be about 30 -fold above basal level. Upon deletion of 11 additional nucleotides, where an EGR-binding region was identified (Figure 2B), we observed an almost 10-fold reduction in the promoter activity of the remaining segment $-33 /+31$, which retained an activity only 4 -fold above the basal level. Removal of 7 additional nucleotides, identified as a putative binding site for the Krueppel-like factor (KLF) and for the bicoid-like homeodomain transcription factor (BCDF; MathInspector - Genomatix; Figure 2B), or a putative binding site for transcription factor II D (TFIID; Figure 2A) (24), further reduced the transcription efficiency to 3-fold above basal level (Figure 3). The transcription of the shortest fragment of the promoter region still occurred, although in a lower level. This agrees with experiments reported by Kiela et al. (25), who showed that the construct spanning $-20+/ 58$ bp exhibited significant activity (4.9-fold above background) in Caco-2 cells.

\section{Point mutations in putative binding elements and GMSA}

As depicted in Figure 4, point mutations in EGR-1/Sp1 elements at -72 and -70 resulted in a significant decrease in promoter activity of the $-85 /+31$ segment. In a series of gel mobility shift assays (GMSAs), a double-stranded oligonucleotide (-80 to -61) spanning the EGR-1/Sp1 motif was used as an end-labeled probe and was incubated with nuclear extracts from OKP cells. We demonstrated that this sequence forms at least two specific shifted DNA-protein complexes (C1-C2; Figure 5A). Protein-DNA interaction in $\mathrm{C} 2$ was less stable, since it was displaced by $5 \mathrm{X}$ molar excess of an unlabeled WT probe, while in C1 this interaction was not totally displaced even by $50 \mathrm{X}$ of unlabeled WT probe. We also tested the ability of individual and composite mutants of the EGR-1/Sp1 binding sites, used in 50X molar excess, to compete for binding as an indirect measure of their affinities for transcription factors. Single site -72 (M1) and -70 (M2) mutants, as well as composite mutants (-72/ -70; M3) were ineffective as competitors, showing the importance of both sites in interacting with transcription factors. The identities of the proteins present in these complexes were established by super shift assays (Figure 5B). The

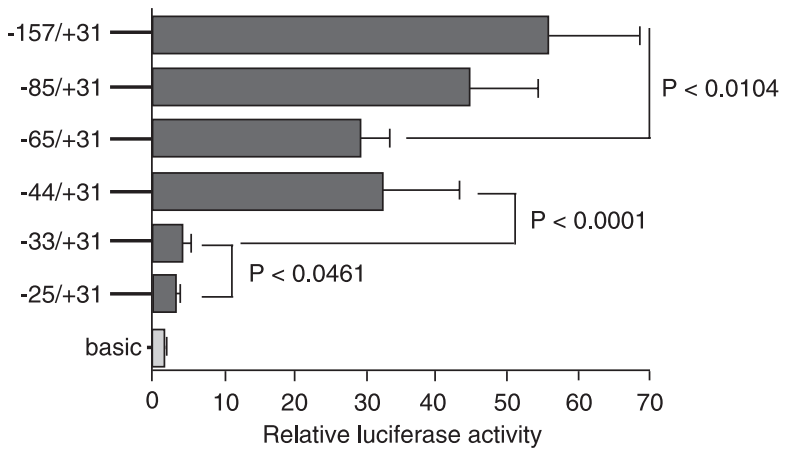

Figure 3. Promoter activity of various 5'-deletion constructs of the proximal promoter region of the rat NHE3 gene. Firefly luciferase reporter constructs containing various lengths of the proximal NHE3 promoter were generated as described. The chimeric promoter-Firefly luciferase gene with different 5 '-ends and a common 3 '-end $(+31)$ was cotransfected with $\mathrm{pRL}$ into opossum kidney, clone P (OKP) cells. After $24 \mathrm{~h}$, the cells were lysed and assayed for Firefly and Renilla luciferase activities. The promoter activity is expressed relative to the activity of the promoterless vector and is normalized for variations in transfection efficiency using Renilla luciferase activity. Data are reported as means \pm SEM for at least three independent transfection experiments performed in triplicate. The spanning region is indicated on the left side of each bar. Statistical analysis was carried out by ANOVA followed by the Tukey test.

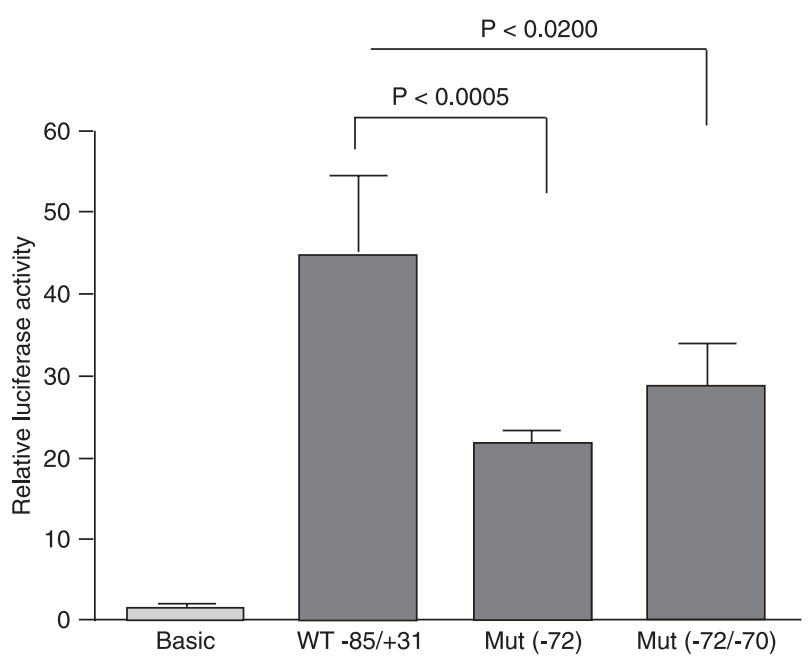

Figure 4. Functional role of the Sp1/EGR-1 binding site at $-72 /$ -66 in the proximal promoter of the NHE3 gene. The promoter activity of the wild-type (WT) and mutated (Mut) segments spanning $-85 /+31$ was evaluated by measuring luciferase activity in transient transfected opossum kidney, clone $P(O K P)$ cells. A single mutation of the -72 nucleotide ( $G$ to $A$ ), or a double mutation of the -70 and -72 nucleotides ( $G$ to $A$ ) were introduced by PCR in the putative binding site to Sp1/EGR-1. The promoter activity is reported relative to the activity of the promoterless vector and is normalized for variations in transfection efficiency using Renilla luciferase activity. Data are reported as means \pm SEM for at least three independent transfection experiments performed in triplicate. Statistical analysis was carried out by ANOVA followed by the Tukey test. 
anti-Sp1 antibody displaced the C2 complex, identifying the protein component of this complex as Sp1. The anti-EGR-1 antibody displaced the $\mathrm{C} 1$ complex, identifying the protein component of this complex as EGR-1, and the anti-Sp3 antibody did not affect any of the DNA-protein complexes. The expression of the transcription factors Sp1, Sp3, and EGR-1 in OKP cells was confirmed by Western blots by using rabbit polyclonal antibodies (Figure $5 \mathrm{C}$ ), also used in super shift assays (Figure $5 \mathrm{~B})$.

When analyzing the $-65 /+31$ segment, we mutated the positions -57 and -58 (CG to AA), at a putative binding site for TBP/Sp1 (Figure 2B; Mathlnspector) or for Sp1 (Figure 2A) (24), and the positions -50 and -51 (GC to $A A$ ), at a putative binding site for AP2 (Figure 2A) (24) or TBP/EGR (Figure 2B) (Mathlnspector). The first two mutations reduced the promoter activity by $63 \%$, and mutations in the four positions simultaneously reduced the promoter activity by $81 \%$, as presented in Figure 6.

A double-stranded oligonucleotide including the pro- moter segment of -61 to $-42 \mathrm{bp}$ was end-labeled and used for EMSA with a nuclear extract of OKP cells. Figure 7A shows that this sequence forms two specific shifted DNAprotein complexes (C1-C2) when incubated with a nuclear extract of OKP cells. The affinity of the binding sites for the transcription factors was indirectly estimated by adding cold WT (5 to $50 \mathrm{X}$ molar excess) or mutated oligonucleotides (50X molar excess) in order to compete for binding sites putatively occupied by labeled probes. The labeled probe was displaced only with the 50X WT probe. The composite mutant $-58 /-57$ probe (M1) efficiently competed for the $\mathrm{C} 1$ complex, but not for the $\mathrm{C} 2$ complex, suggesting that $\mathrm{C} 2$ binds to nucleotides $-58 /-57$ (CG). The composite mutant $-51 /-50$ (M2) efficiently competed for both complexes, suggesting that nucleotides at positions $-51 /-50$ are not relevant for the binding of these complexes. Interestingly, the composite mutant -58/-57/-51/-50 (M3) efficiently competed for both complexes, suggesting that mutations at positions $-51 /-50$ made the mutations at positions $-58 /-57$

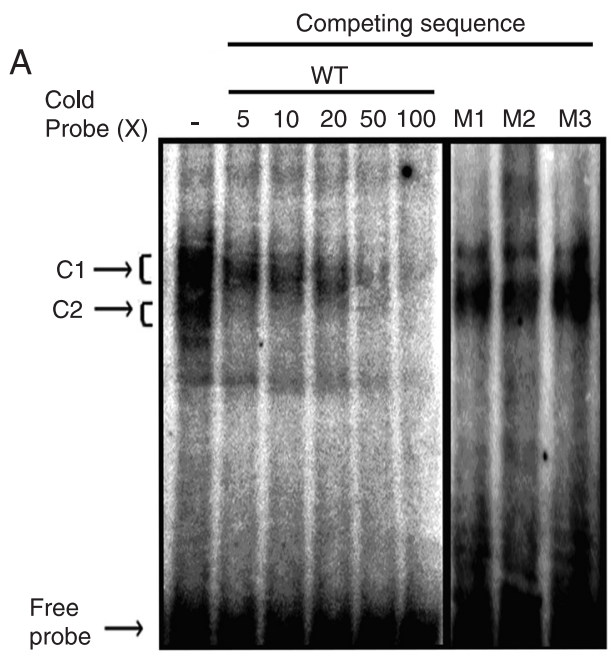

WT CGCAGCGGGGGCGGAAGGC

M1 TTCACGTGCGCA GCGGAGGCGGGAAGGCCC (-72) M2 TTCACGTGCGCA GCGGGGACGGGAAGGCCC (-70) M3 TTCACGTGCGCAGCGGAGACGGGAAGGCCC (-72/-70)
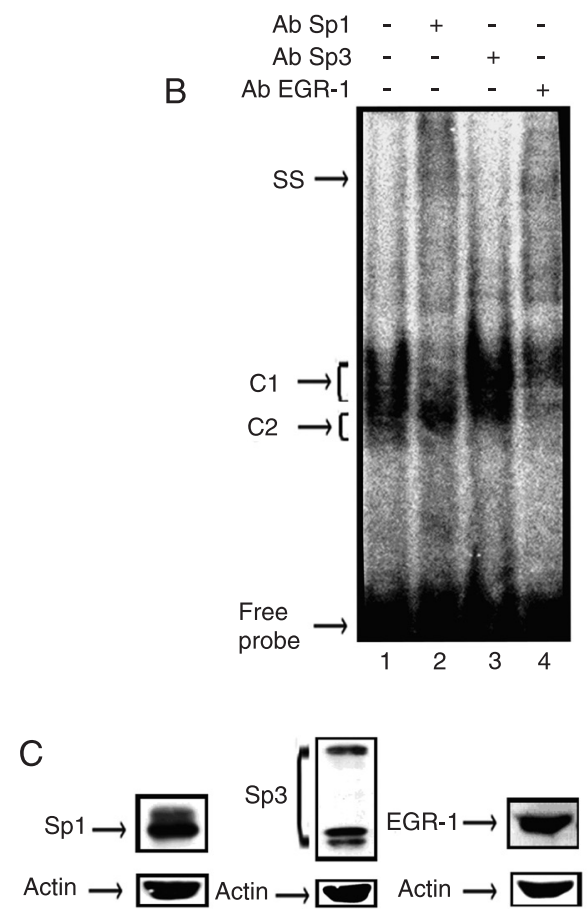

Figure 5. Identification of proteins binding to the region -80/-61 of the NHE3 gene promoter. $A$, The -80/-61-bp sequence used as a probe in a gel mobility shift assay formed at least two specific shifted DNA-protein complexes (C1 and C2). These complexes competed in a dose-dependent manner with the wild-type (WT) unlabeled probe. Single site mutants -72 (M1), -70 (M2), and a composite mutant (M3) could not compete for these complexes. The cis-elements are emphasized by rectangles and the bold and underlined letters indicate mutations introduced. $B$, In a gel mobility super shift assay formed a specific complex with an opossum kidney clone $\mathrm{P}$ (OKP) nuclear extract (lane 1), specific protein/DNA complexes were disassembled with anti-Sp1 (C1; lane 2), and anti-EGR-1 antibodies (C2; lane 4). The Sp3 antibody did not disturb the complexes (lane 3). C, Western blot analyses of total proteins of OKP cells were performed using antibody against Sp1, Sp3 and EGR-1. Analyses of actin expression were used as an internal control. 
acceptable as competitors for complex C2, probably due to a conformational change. These results might indicate low specificity of the selected sites for the transcription factors. It would be important to know the sequence of the $D$. virginiana promoter, which is not available at GenBank, in

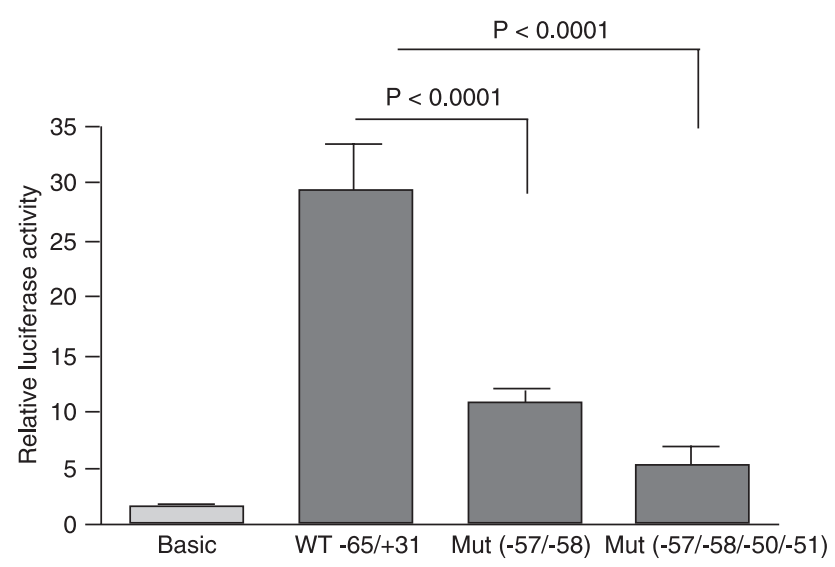

A

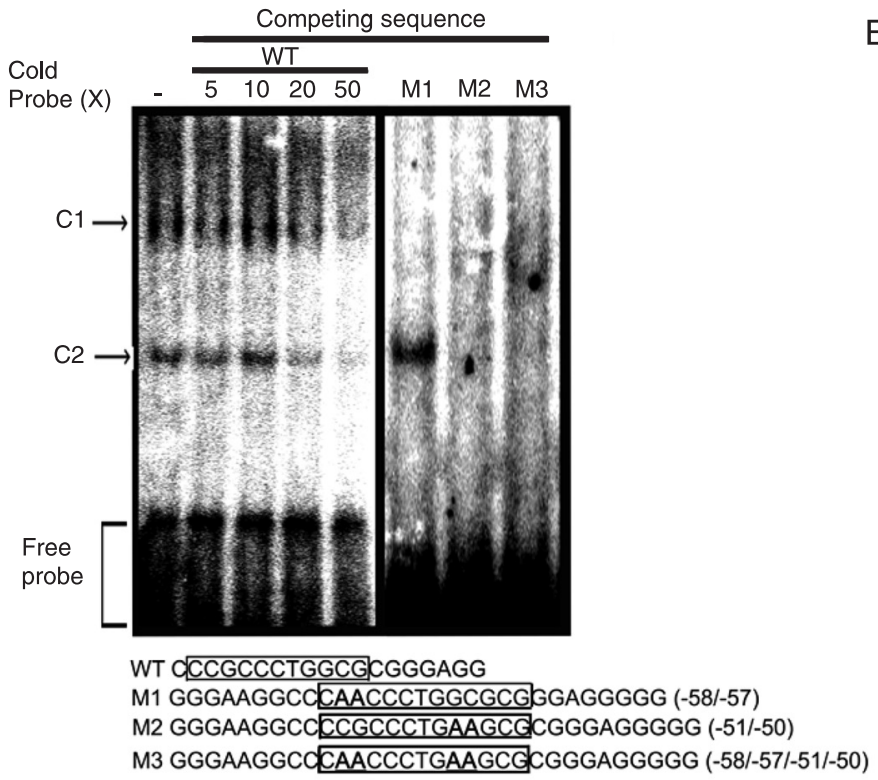

order to obtain a better prediction of the consensus sites for transcription factors in this species. In super shift assays, all the antibodies used, anti-Sp1, anti-Sp3 and anti-EGR-1, displaced the $\mathrm{C} 2$ complex (Figure 7B), suggesting that any one of the analyzed transcription factors could participate in

Figure 6. Functional role of Sp1/TBP and AP2/EGR-1 binding sites in the proximal promoter of the NHE3 gene. The promoter activity of the wild-type (WT) and mutated (Mut) segments spanning $-65 /+31$ was evaluated by measuring luciferase activity in transient transfected opossum kidney, clone $P(O K P)$ cells. Double mutations of the -58 and -57 nucleotides (CG to AA), and combined double mutations of the $-58 /-57$ and $-51 /-50$ nucleotides (GC to $A A$ ) were introduced by PCR into the putative binding sites to Sp1/TBP and AP2/EGR-1. The promoter activity is expressed relative to the activity of the promoterless vector and is normalized for variations in transfection efficiency using Renilla luciferase activity. Data are reported as means \pm SEM for at least three independent transfection experiments performed in triplicate. Statistical analysis was carried out by ANOVA followed by the Tukey test.

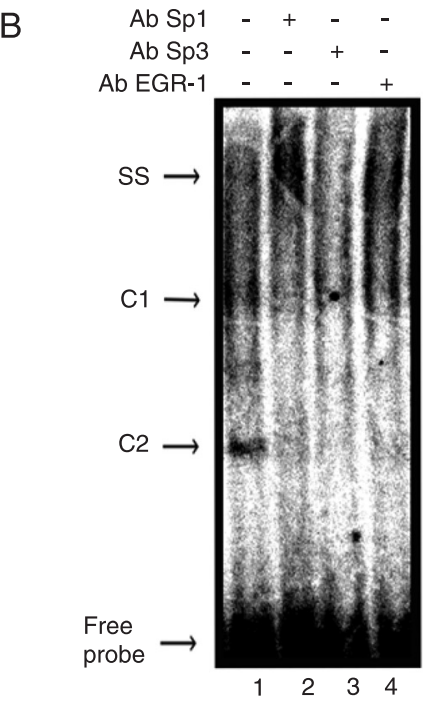

Figure 7. Identification of proteins binding to the -61/-42 region of the NHE3 gene promoter. $A$, The -61/-42bp sequence used as probe in a gel mobility shift assay formed two specific shifted DNA-protein complexes (C1 and $\mathrm{C} 2$ ) with opossum kidney clone $\mathrm{P}(\mathrm{OKP})$ nuclear proteins. These complexes competed in a dosedependent manner with the unlabeled wild-type (WT) probe. The composite mutant -58/-57 probe (M1) efficiently competed for the $\mathrm{C} 1$ complex, but not for the $\mathrm{C} 2$ complex. The composite mutant $-51 /-50$ (M2) as well as the composite mutant -58/-57/-51/-50 (M3) efficiently competed for both complexes. The cis-elements are emphasized by rectangles and the bold and underlined letters indicate mutations. $B$, In the gel mobility super shift assay formed a specific complex with OKP nuclear extract (lane 1), the C2 complex was disassembled when any one of the three antibodies, anti-Sp1, -Sp3 and -EGR-1, was added to the OKP nuclear extract incubation mix (lanes 2, 3, and 4). Apparently a super shift can be observed in lane 2, and possibly in lane 4, indicating that Sp1 and perhaps EGR-1 participates in these complexes. 
the DNA-protein complex. Apparently a super shift can be observed in lane 2, and possibly in lane 4, indicating that Sp1 and perhaps EGR-1 participate in these complexes, although these results are not conclusive.

\section{Discussion}

The effective role of some regulatory elements in the rat 5'FS and in the human NHE3 gene in the modulation of transcription has been reported mainly in human intestinal cell lines (Caco-2, C2BBel) (17-20,26). Renal proximal tubule (OKP and LLC-PK1) $(9,12,13,21)$ and collecting duct cells (MDCK) (27) have also been used for analysis of the NHE3 promoter, although less frequently. OKP, a well-established cell culture, is a good proximal tubule model for studying NHE3 because, in addition to having a relatively stable phenotype, NHE3 is virtually the only $\mathrm{Na}^{+} / \mathrm{H}^{+}$antiporter present in these cells, and many of the signaling molecules relevant for the regulation of its expression and activity are also expressed in OKP cells. Moreover, we have shown in the present study that endogenous opossum transcription factors bind with high or moderate affinity to DNA elements of the rat NHE3 promoter, suggesting a high level of identity between the DNA binding domains of opossum transcription factors and those of the corresponding transcription factors of the rat. Although recombinant exogenous transcription factors have been expressed in OKP cells in order to evaluate protein-DNA interactions by gel shift assays (21), the interaction between opossum proteins and rat NHE3 promoter had not been clearly demonstrated to date.

By performing the sequential shortening of the 5'-end of the NHE3 gene regulatory region, we observed that the segment spanning the first $152 \mathrm{bp}$ upstream of the transcription start site was more efficient in promoting the reporter gene expression than the other segments extending further upstream. The most relevant elements for NHE3 gene transcription are included in the first 150 bp of the 5'FS. However, a careful analysis of the transcriptional activity of the different segments of the 5'FS demonstrated that inhibitory (-1196/-889 and $-467 /-315)$ and stimulatory (-889/-467) elements can fine tune NHE3 expression. Conspicuous differences between the activity of different segments of the rat NHE3 promoter spanning regions $-1360 /+58,-1194 /+58,-715 /+58$, and $-450 /+58$ were not observed in Caco cells, and shorter fragments such as $-320 /+58$ and $-81 /+58$ were less active than the larger ones (25). These results contrast with our findings. Excluding differences in transfection efficiency, which we avoided by careful quantification of the constructs and normalization to an internal control, our results suggest a considerable flexibility in NHE3 expression in renal proximal tubules, depending on which regions of the promoter are available for interaction with transcription factors. In vivo, the availability of different regions of the promoter depends on the chromatin structure, which is dynamic and can change depending on the interacting factors.

We localized the most relevant elements for efficient NHE3 gene transcription between -85 and -33 of 5 'FS. Analyses of the activity of the human NHE3 proximal promoter in intestinal cells after sequential shortening at the 5 '-end have shown results not substantially different from ours (20). The segment spanning $-95 /+5$ exhibited maximum activity; the segment spanning $-43 /+5$, which approximately corresponds to our rat segment $-33 /+31$, was no more than 3 -fold as active as the promoterless construct (17).

Through the analysis of the rat NHE3 gene promoter by MathInspector we identified in the region -85 to -33 putative binding sites for CEBP/EBOX, Sp1 (2 cis-elements), EGR1, NF-KB, E2F-Myc, and 2 TBP-dependent core promoter elements for RNA polymerase II transcription from the TATA-less promoter. Some, but not all, of these identified cis-elements have already been described (12). There is a high level of similarity between the rat and human NHE3 gene promoters in the first $100 \mathrm{bp}$ upstream to the start site, and the consensus sequences in the segment spanning -85 to -33 are well conserved between the two species (17), strongly indicating that this region is essential for NHE3 expression. Single (-72 nucleotide) or double (-72 and -69 nucleotides) mutations in the Sp1 (Sp1A) or EGR-1/E2FMyc consensus sequence significantly reduced the promoter activity of the segment spanning -85 to +31 .

The gel shift experiments with the probe spanning -80 to -61 confirmed that in OKP cells, under basal conditions, Sp1 and EGR-1, but not Sp3, bind to this region. The observed interaction of Sp1 and EGR-1 with the corresponding binding sites is in accordance with the role of this region for the activation of the human NHE3 gene transcription by PMA and $\mathrm{NaB}$, and inhibition by IFN- $/$ /TNF- $\alpha(18-20)$ in intestinal cells. PMA favors EGR-1 to the detriment of Sp1/Sp3 binding, and IFN- $\mathrm{Y} / \mathrm{TNF}-\alpha$ down-regulate the expression of human NHE3 by cAMP-dependent protein kinase (PKA)-mediated phosphorylation of Sp1 and Sp3 transcription factors (18). Previous results from our laboratory demonstrating the PKA-dependent inhibitory effect of parathyroid hormone on the proximal NHE3 promoter in OKP cells (15), an effect that might be related to phosphorylation of $\mathrm{Sp} 1$, are also additional evidence for the relevance of this Sp1 binding for efficient expression of NHE3.

The Sp1 binding site previously defined as SpB by Kiela et al. (26) was also shown to be relevant for NHE3 expression in proximal tubule cells. Mutations in positions -58/-57 or in positions $-58 /-57$ and $-51 /-50$, where cis-elements for Sp1/TBP/Myc and EGR-1/AP2 were identified, respectively, severely affected the promoter activity of the $-65 /+31$ segment. However, complete removal of the 21 nucleotide from -65 to -44 did not change the expression of the reporter gene. This may be due to conformational changes in the mutated DNA that disturbed relevant protein interactions with sites downstream from $-44 \mathrm{bp}$, although these interactions were not disturbed when the whole segment of 21 
nucleotides was removed. We could not clearly establish which transcription factor interacts with the -61 to -42 region. Antibodies to Sp1, Sp3 and EGR-1 all displace the C2 complex. This is a region where binding sites for all these transcription factors overlap, and binding of an antibody to any one of these transcription factors could destabilize the complex. Apparently, incubation with Sp1 antibody and, less clearly, with EGR-1 antibody resulted in a super shift of DNA/protein complexes, suggesting the participation of Sp1 and perhaps EGR-1 in these complexes.

The key element for the stability of the primary transcription factors appears to be in the next downstream 11 bp, where an EGR-1 binding site was identified, since its removal resulted in an almost 10 -fold decrease in promoter activity.

Taken together, our results support the conclusion that essential regulatory elements for the expression of NHE3 in the proximal promoter (initial 150 nucleotides of 5'FS)

\section{References}

1. Wang T, Hropot M, Aronson PS, Giebisch G. Role of NHE isoforms in mediating bicarbonate reabsorption along the nephron. Am J Physiol Renal Physiol 2001; 281: F1117F1122.

2. Aronson PS. Ion exchangers mediating $\mathrm{NaCl}$ transport in the renal proximal tubule. Cell Biochem Biophys 2002; 36: 147-153.

3. Alexander $\mathrm{RT}$, Grinstein $\mathrm{S}$. $\mathrm{Na}^{+} / \mathrm{H}^{+}$exchangers and the regulation of volume. Acta Physiol 2006; 187: 159-167.

4. Krapf R, Pearce D, Lynch C, Xi XP, Reudelhuber TL, Pouyssegur $\mathrm{J}$, et al. Expression of rat renal $\mathrm{Na} / \mathrm{H}$ antiporter mRNA levels in response to respiratory and metabolic acidosis. $J$ Clin Invest 1991; 87: 747-751.

5. Moe OW, Miller RT, Horie S, Cano A, Preisig PA, Alpern RJ. Differential regulation of $\mathrm{Na} / \mathrm{H}$ antiporter by acid in renal epithelial cells and fibroblasts. J Clin Invest 1991; 88: 17031708.

6. Amemiya M, Yamaji Y, Cano A, Moe OW, Alpern RJ. Acid incubation increases NHE-3 mRNA abundance in OKP cells. Am J Physiol 1995; 269: C126-C133.

7. Fisher KA, Lee SH, Walker J, leto-Fang C, Ginsberg L, Stapleton SR. Regulation of proximal tubule sodium/hydrogen antiporter with chronic volume contraction. Am J Physiol Renal Physiol 2001; 280: F922-F926.

8. Dixit MP, Xu L, Xu H, Bai L, Collins JF, Ghishan FK. Effect of angiotensin-II on renal $\mathrm{Na}^{+} / \mathrm{H}^{+}$exchanger-NHE3 and NHE2. Biochim Biophys Acta 2004; 1664: 38-44.

9. Xu L, Dixit MP, Nullmeyer KD, Xu H, Kiela PR, Lynch RM, et al. Regulation of $\mathrm{Na}^{+} / \mathrm{H}^{+}$exchanger-NHE3 by angiotensin-II in OKP cells. Biochim Biophys Acta 2006; 1758: 519-526.

10. Klisic J, Hu MC, Nief V, Reyes L, Fuster D, Moe OW, et al. Insulin activates $\mathrm{Na}(+) / \mathrm{H}(+)$ exchanger 3: biphasic response and glucocorticoid dependence. Am J Physiol Renal Physiol 2002; 283: F532-F539.

11. Collins JF, Xu H, Kiela PR, Zeng J, Ghishan FK. Functional and molecular characterization of NHE3 expression during ontogeny in rat jejunal epithelium. Am J Physiol 1997; 273: are similarly used in intestinal and in renal proximal tubule cells. However, considerable differences in the fine-tuning modulation by regulatory elements upstream to -150 may be suggested, in view of the variability in the promoter efficiency depending on the length of the analyzed 5'FS. Twenty to twenty-five nucleotides of 5'FS are the minimum necessary for the assembly of primary transcription factors, but an efficient transcription only occurs with 44 bp upstream to the start site. We demonstrated that regulatory elements present in the segment spanning -85 to -33 , including binding sites for Sp1 and EGR-1, are relevant modulators of NHE3 gene transcription under basal, non-stimulated conditions, in renal proximal tubules.

\section{Acknowledgments}

Research supported by FAPESP, CNPq, and CAPES.

\section{C1937-C1946.}

12. Kandasamy RA, Orlowski J. Genomic organization and glucocorticoid transcriptional activation of the rat $\mathrm{Na}^{+/} \mathrm{H}^{+}$ exchanger Nhe3 gene. J Biol Chem 1996; 271: 1055110559.

13. Cano A, Baum M, Moe OW. Thyroid hormone stimulates the renal $\mathrm{Na} / \mathrm{H}$ exchanger $\mathrm{NHE} 3$ by transcriptional activation. Am J Physiol 1999; 276: C102-C108.

14. Girardi AC, Titan SM, Malnic G, Reboucas NA. Chronic effect of parathyroid hormone on NHE3 expression in rat renal proximal tubules. Kidney Int 2000; 58: 1623-1631.

15. Bezerra CN, Girardi AC, Carraro-Lacroix LR, Reboucas NA. Mechanisms underlying the long-term regulation of NHE3 by parathyroid hormone. Am J Physiol Renal Physiol 2008; 294: F1232-F1237.

16. Cano A. Characterization of the rat NHE3 promoter. Am J Physiol 1996; 271: F629-F636.

17. Malakooti J, Memark VC, Dudeja PK, Ramaswamy K. Molecular cloning and functional analysis of the human $\mathrm{Na}(+) / \mathrm{H}(+)$ exchanger NHE3 promoter. Am J Physiol Gastrointest Liver Physiol 2002; 282: G491-G500.

18. Amin MR, Malakooti J, Sandoval R, Dudeja PK, Ramaswamy K. IFN-gamma and TNF-alpha regulate human NHE3 gene expression by modulating the $\mathrm{Sp}$ family transcription factors in human intestinal epithelial cell line C2BBe1. Am J Physiol Cell Physiol 2006; 291: C887-C896.

19. Amin MR, Dudeja PK, Ramaswamy K, Malakooti J. Involvement of Sp1 and Sp3 in differential regulation of human NHE3 promoter activity by sodium butyrate and IFN-gamma/ TNF-alpha. Am J Physiol Gastrointest Liver Physiol 2007; 293: G374-G382.

20. Malakooti J, Sandoval R, Amin MR, Clark J, Dudeja PK, Ramaswamy K. Transcriptional stimulation of the human NHE3 promoter activity by PMA: PKC independence and involvement of the transcription factor EGR-1. Biochem $J$ 2006; 396: 327-336.

21. Cole JA, Forte LR, Krause WJ, Thorne PK. Clonal sublines 
that are morphologically and functionally distinct from parental OK cells. Am J Physiol 1989; 256: F672-F679.

22. Laemmli UK. Cleavage of structural proteins during the assembly of the head of bacteriophage T4. Nature 1970; 227 : 680-685.

23. Girardi AC, Knauf F, Demuth HU, Aronson PS. Role of dipeptidyl peptidase IV in regulating activity of $\mathrm{Na}^{+} / \mathrm{H}^{+}$exchanger isoform NHE3 in proximal tubule cells. Am J Physiol Cell Physiol 2004; 287: C1238-C1245.

24. Rohman MS, Emoto N, Nonaka H, Okura R, Nishimura M, Yagita $\mathrm{K}$, et al. Circadian clock genes directly regulate expression of the $\mathrm{Na}(+) / \mathrm{H}(+)$ exchanger NHE3 in the kidney. Kidney Int 2005; 67: 1410-1419.
25. Kiela PR, LeSueur J, Collins JF, Ghishan FK. Transcriptional regulation of the rat NHE3 gene. Functional interactions between GATA-5 and Sp family transcription factors. $J$ Biol Chem 2003; 278: 5659-5668.

26. Kiela PR, Hines ER, Collins JF, Ghishan FK. Regulation of the rat NHE3 gene promoter by sodium butyrate. Am J Physiol Gastrointest Liver Physiol 2001; 281: G947-G956.

27. Su HW, Wang SW, Ghishan FK, Kiela PR, Tang MJ. Cell confluency-induced Stat3 activation regulates NHE3 expression by recruiting Sp1 and Sp3 to the proximal NHE3 promoter region during epithelial dome formation. Am J Physiol Cell Physiol 2009; 296: C13-C24. 